\title{
Tracking of physical fitness levels from childhood and adolescence to adulthood: a systematic review and meta-analysis
}

\author{
Antonio García-Hermoso^, Mikel Izquierdo^, Robinson Ramírez-Vélez^ \\ Navarrabiomed, Hospital Universitario de Navarra (HUN), Universidad Pública de Navarra (UPNA), IdiSNA, Pamplona, Spain \\ Contributions: (I) Conception and design: A García-Hermoso; (II) Administrative support: None; (III) Provision of study materials or patients: \\ None; (IV) Collection and assembly of data: A García-Hermoso, R Ramírez-Vélez; (V) Data analysis and interpretation: A García-Hermoso; (VI) \\ Manuscript writing: All authors; (VII) Final approval of manuscript: All authors. \\ Correspondence to: Antonio García-Hermoso, PhD. Navarrabiomed, Hospital Universitario de Navarra (HUN), Universidad Pública de Navarra \\ (UPNA), IdiSNA, Pamplona, Spain. Email: antonio.garciah@unavarra.es.
}

Background: Prospective and large studies indicate that high physical fitness levels during young are
beneficial for health during adulthood. The aim of the study was to investigate the tracking of physical fitness
components from childhood and/or adolescence to adulthood.
Methods: Two authors systematically searched MEDLINE and Web of Science electronic databases for
relevant articles. Studies with apparently healthy youth aged 6-18 years who track their physical fitness to
adulthood were included. Our study carried out according to the Preferred Reporting Items for Systematic
Reviews and Meta-Analyses (PRISMA). Correlation coefficients (r) were used as effect size. Random-effects
models were used to estimate the pooled effect size. Correlation coefficients were interpreted as follows:
$<0.30$ low stability, 0.30 to 0.60 moderate stability, and $>0.60$ high stability. Risk of bias of each study was
determined by The Quality Assessment Tool for Observational Cohort and Cross-sectional Studies. Results: Twenty-one prospective studies were included in the meta-analysis ( $\mathrm{n}=6,197$ participants at follow-up, 47.4\% women). The mean length of follow-up was 20.8 years. Overall, cardiorespiratory fitness $\left(\mathrm{r}=0.38 ; 95 \%\right.$ CI: 0.29-0.48; $\left.\mathrm{I}^{2}=92.7 \%\right)$, muscular strength $\left(\mathrm{r}=0.51 ; 95 \% \mathrm{CI}: 0.43-0.59 ; \mathrm{I}^{2}=87.9 \%\right)$, and muscular endurance ( $\mathrm{r}=0.50 ; 95 \%$ CI: $0.36-0.86 ; \mathrm{I}^{2}=94.5 \%$ ) show moderate tracking from childhood and/ or adolescence to adulthood, independent of test used and length of follow-up. This moderate tracking was slightly stronger in women than in men and from adolescence compared to childhood. Trunk flexibility component, assessed with the sit and reach test, exhibits high stability (r=0.69; 95\% CI: 0.58-0.81; $\left.\mathrm{I}^{2}=92.9 \%\right)$. Interestingly, meta-regression analysis shows positive association between correlation coefficient for flexibility and the length of follow-up ( $\beta=0.017$; 95\% CI: $0.012-0.021)$.

Discussion: Although the current study found inconsistency between results, the findings suggest that acquiring high physical fitness levels should be targeted already from childhood and adolescence given that low levels of fitness in adulthood are related with several chronic diseases and mortality.

Trial Registration: Registration number CRD42021279143.

Keywords: Stability; muscular fitness; cardiorespiratory fitness; flexibility

Submitted Oct 26, 2021. Accepted for publication Feb 15, 2022.

doi: $10.21037 / \mathrm{tp}-21-507$

View this article at: https://dx.doi.org/10.21037/tp-21-507

\footnotetext{
^ ORCID: Antonio García-Hermoso, 0000-0002-1397-7182; Mikel Izquierdo, 0000-0002-1506-4272; Robinson Ramírez-Vélez, 00000003-3075-6960.
} 


\section{Introduction}

A growing body of epidemiological and clinical evidence has examined the relationship between physical fitness and health outcomes across the life course. Acquiring and maintaining satisfactory fitness levels throughout life seem to be important, since these components are related with protection from risks to develop several chronic diseases. Overall, findings in adults show that greater physical fitness, mainly cardiorespiratory endurance and muscular fitness, is associated with reduced all-cause mortality $(1,2)$ and risk of developing a wide range of non-communicable diseases (3), independent of potential confounding variables. In the same way, evidence in the scientific literature highlights the importance of acquiring adequate levels of the components of physical fitness from early years (4) since these are related to better body composition and a healthier cardiometabolic profile later in life $(5,6)$.

Tracking refers to the maintenance of relative position within a group over time for at least two points in time (7). Longitudinal data for physical fitness through childhood, adolescence, and adulthood are not fully known yet. Differences in the type of physical fitness tests, follow-up, the initial age at baseline (i.e., childhood or adolescence), and different statistical models taken, make difficult to draw any conclusions. Generally interage show higher stability than for indicators of physical activity (7). Seems to be important to identify risk factors from childhood and adolescence for low physical fitness later in adulthood to offer solutions and prevent an unhealthy deterioration (8).

As explained earlier, given the importance of childhood and adolescence fitness relative to subsequent adult health outcomes, it is valuable to examine how comprehensive fitness components track throughout childhood and/or adolescence to adulthood. To explore if targeting childhood and adolescence physical fitness could help improve future health, a meta-analytic approach quantifying how levels of physical fitness track across the life course is needed. Therefore, the aim of the study was to determine the tracking of physical fitness components from childhood and/or adolescence to adulthood. We present the following article in accordance with the PRISMA reporting checklist (available at https://tp.amegroups.com/article/ view/10.21037/tp-21-507/rc).

\section{Methods}

The protocol was registered in PROSPERO (the international prospective register of systematic reviews) (CRD42021279143). Each part of the systematic review (i.e., literature selection, data extraction) was performed by two authors (AGH \& RRV) and disagreements were resolved through consultation with a third author (MI).

\section{Selection criteria}

Longitudinal cohort studies that measured physical fitness in childhood and/or adolescence (ages 6-18 years) and its prospective association in adulthood (age 20 years and over) were eligible. Any measure of physical fitness was considered. Where multiple studies reported on the same participants (i.e., same cohort), the paper judged to contain recent data or when all data results are available, was used.

\section{Information sources and search strategy}

MEDLINE and Web of Science electronic databases were searched for articles from inception to 5 October 2021. An example of electronic search strategy used in MEDLINE was as follows: $((((()((($ physical fitness) OR (muscular strength)) OR (muscular fitness)) OR (cardiorespiratory fitness)) AND (aerobic fitness)) OR (flexibility)) AND (tracking)) OR (stability)) AND (cohort)) AND (longitudinal)) AND (prospective). Additionally, searches strategies were limited to children and adolescents aged 6-18 years.

\section{Selection process and data collection process}

Two authors independently obtained the following data from each article: (I) study characteristics (authors and publication year, sample size, sex, age of individuals, country, years of follow-up); (II) physical fitness details [test used, component of physical fitness (i.e., cardiorespiratory fitness, muscular endurance, muscular strength, and/or flexibility)]; and (III) analysis and results.

\section{Risk of bias in individual studies}

Quality Assessment Tool for Observational Cohort and Cross-sectional Studies (9) was used to determine the quality of each study. This scale is comprised of 14 items classified as "yes", "no" or "not reported".

\section{Statistical analysis}

The effect sizes reported by studies were correlations 
coefficients (r). DerSimonian-Laird random-effects inversevariance model (10) was used using the Stata 17.0 (StataCorp, College Station, TX, USA). According to Malina (11), coefficient of correlation can be interpreted as follows: $<0.30$ low stability, 0.30 to 0.60 moderate stability, and $>0.60$ high stability. We considered a $\mathrm{P}$ value of $<0.05$ to be statistically significant.

It is important to clarify the following aspects relating to our statistical analyses: (I) pooled analysis (i.e., meta-analyses) were only performed for fitness parameters that were included in three or more articles; (II) when different sexes were included in a study, their data were analyzed independently; (III) when two or more tests for measuring the same fitness components (e.g., muscular strength) were included in an article, we included the higher correlation coefficient; and (IV) data from multiple articles of the same cohort were extracted and reported as a particular study, except there was no overlap.

\section{Synthesis of results}

For each analysis, heterogeneity across correlation coefficients was determined using the total variance and the inconsistency index $\left(\mathrm{I}^{2}\right)(12) . \mathrm{I}^{2}$ values of $<25 \%, 25-75 \%$, and $>75 \%$ were classified as small, moderate, and high heterogeneity, respectively (13).

\section{Risk of bias across studies}

The Luis Furuya-Kanamori (LFK) index was used to examine small-study effects and publication bias, respectively. An LFK value $>1$ or less than $<-1$ suggests minor asymmetry and a value greater $>2$ or $<-2$ suggests major asymmetry (14).

\section{Additional analysis}

Whenever possible, sub-group analysis according to sex, physical fitness test, and age at baseline (i.e., childhood or adolescence) were examined. Finally, random-effects metaregression (DerSimonian and Laird model) was conducted to determine whether length of follow-up (years) was a moderator in these associations, showing the unstandardized regression coefficients $(\beta)$.

\section{Results}

\section{Study selection}

Through database searching we identified 7,044 records. Of these, 5,635 studies were excluded by duplication. We screened 28 full-text articles and seven was excluded.
The reasons for exclusion were inappropriate study population (three studies) (15-17) or duplicate results (four studies) (18-21). In total, 21 studies met the inclusion criteria, but only 19 were included in the meta-analysis due to missing data in two of them. The PROSPERO flow diagram is shown in Figure 1.

\section{Study characteristics}

Our study included a total of 6,197 participantes $(47.4 \%$ women). All articles included men and women with the exception of two studies that included only men $(22,23)$ and another only women (24). Sample sizes ranged from 45 (25) to 822 (26) and length of follow-up from 7 (27) to 45 years (28) (mean: 20.8 years). Studies tracked the fitness components from childhood (29-31), adolescence (22-25,27,32-40), or both $(26,28,41,42)$ to adulthood (Table 1$)$.

\section{Physical fitness measurement}

Cardiorespiratory fitness was mainly determined with laboratory tests [e.g., maximal $(23,25,28,32-34,36,38,39)$ or submaximal (37) test on a cycloergometer or treadmill with spirometry to determine oxygen consumption $\left(\mathrm{VO}_{2}\right)$, and the physical work capacity at a heart rate of 150 (42) or 170 beats per minute cycloergometer test (29)] or field tests [e.g., 20-m shuttle run test $(22,27), 9-$ min run test $(31,35)$, 12-min endurance test (40), 50-m shuttle run (24)] (Table 1).

Regarding muscular fitness (Table 1), most of the studies assessed muscular strength using the handgrip test $(23,25,26,29,30,35,41)$ and explosive test such as the standing long jump (41), vertical jump (22-24,40), or sargent jump (37). Muscular endurance was assessed using mainly the sit-up test $(23,25,29,31,37)$ and bent arm hang test $(22-24,40)$. Other studies used an isometric strength score (38) and other tests different from those mentioned (Table 1).

Finally, flexibility was assessed using the sit and reach test in all included studies.

\section{Risk of bias within studies}

All studies met at least eight criteria (between 8 and 11 criteria) with an average of 8.8 . Overall, studies could be considered to have low-moderate risk of bias. All 21 studies satisfied items $1,2,4,6,7,8$, and 11 . In contrast, only eight studies fulfilled item 3 (regarding participation rate), six item 10 (regarding exposure assessed more than once over time), 


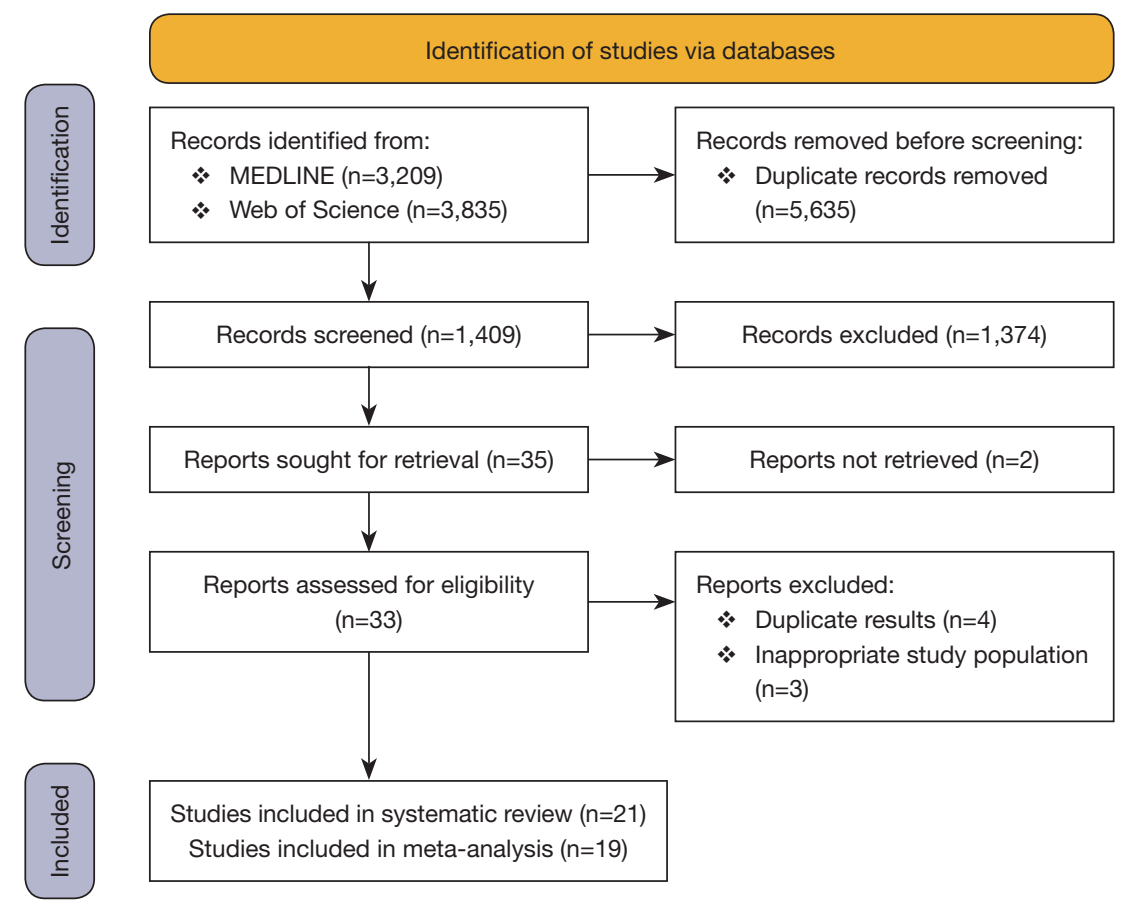

Figure 1 PRISMA flow diagram.

four item 13 (regarding loss to follow-up), and no studies fulfilled items 5 (regarding sample size justification) and 14 (regarding potential confounding) (Table 2).

\section{Meta-analysis}

\section{Cardiorespiratory fitness}

Our meta-analysis shows that cardiorespiratory fitness has moderate track from childhood and/or adolescence to adulthood ( $\mathrm{r}=0.38$; 95\% CI: 0.29-0.48; $\mathrm{I}^{2}=92.7 \%$ ) (Figure 2), with similar results according to sex (men: $\mathrm{r}=0.31,95 \% \mathrm{CI}$ : 0.24-0.39, I $\mathrm{I}^{2}=51.2 \%$; women: $\mathrm{r}=0.40,95 \%$ CI: $0.34-0.45$, $\mathrm{I}^{2}=9.9 \%$ ), test used (maximal cycloergometer test: $\mathrm{r}=0.49$, 95\% CI: $0.33-0.64, \mathrm{I}^{2}=92.0 \%$; maximal treadmill test: $\mathrm{r}=0.33$, 95\% CI: $0.23-0.42, \mathrm{I}^{2}=0 \%$ ), and age at baseline (childhood: $\mathrm{r}=0.38,95 \%$ CI: $0.26-0.49, \mathrm{I}^{2}=27.8 \%$; adolescence: $\mathrm{r}=0.41$, 95\% CI: $0.27-0.54, \mathrm{I}^{2}=93.7 \%$ ).

The meta-regression analysis showed that the length of follow-up did not moderate this association $(\beta=-0.001$; 95\% CI: -0.010 to 0.009 ; $\mathrm{P}=0.863$ ).

Major asymmetry suggestive of small-study effects was observed $($ LKF index $=-4.04)$.

The sensitivity analyses showed no modifications in the results after removing one article at a time.

\section{Muscular fitness}

Our meta-analysis shows that muscular strength has moderate track from childhood and/or adolescence to adulthood ( $\mathrm{r}=0.51$; 95\% CI: $0.43-0.59 ; \mathrm{I}^{2}=87.9 \%$ ) (Figure 2), showing similar stability by sex (men: $r=0.49,95 \% \mathrm{CI}$ : $0.42-0.57, \mathrm{I}^{2}=53.8 \%$; women: $\mathrm{r}=0.51,95 \%$ CI: $0.39-0.62$, $\mathrm{I}^{2}=77.5 \%$ ), test used (leg extensors: $\mathrm{r}=0.57,95 \%$ CI: $0.35-$ $0.79, \mathrm{I}^{2}=96.5 \%$; bench press: $\mathrm{r}=0.46,95 \%$ CI: $0.35-0.56$, $\mathrm{I}^{2}=63.5 \%$; handgrip: $\mathrm{r}=0.48,95 \%$ CI: $0.41-0.56, \mathrm{I}^{2}=74.5 \%$; vertical jump: $\mathrm{r}=0.58,95 \% \mathrm{CI}$ : $\left.0.36-0.80, \mathrm{I}^{2}=96.1 \%\right)$, and age at baseline (childhood: $\mathrm{r}=0.46,95 \%$ CI: $0.41-0.51, \mathrm{I}^{2}=4.1 \%$; adolescence: $r=0.52$, 95\% CI: $0.42-0.61, I^{2}=89.6 \%$ ).

The meta-regression analysis showed that the length of follow-up did not moderate this association ( $\beta=0.004 ; 95 \%$ CI: -0.006 to $0.015 ; \mathrm{P}=0.414$ ).

Minor asymmetry suggestive of small-study effects was observed (LKF index $=-1.10)$.

The sensitivity analyses indicated no modifications in the results after removing 1 study at a time in muscular strength.

Muscular endurance has moderate track from youth to adulthood (r=0.50; 95\% CI: 0.36-0.86; $\mathrm{I}^{2}=94.5 \%$ ) (Figure 2), independent of sex (men: $\mathrm{r}=0.33,95 \%$ CI: $0.20-0.47$, $\mathrm{I}^{2}=55.4 \%$; women: $\mathrm{r}=0.46,95 \%$ CI: $0.32-0.60, \mathrm{I}^{2}=28.6 \%$ ). 
Table 1 Studies that analyzed the tracking of fitness from childhood or adolescence to adulthood or mid-adulthood

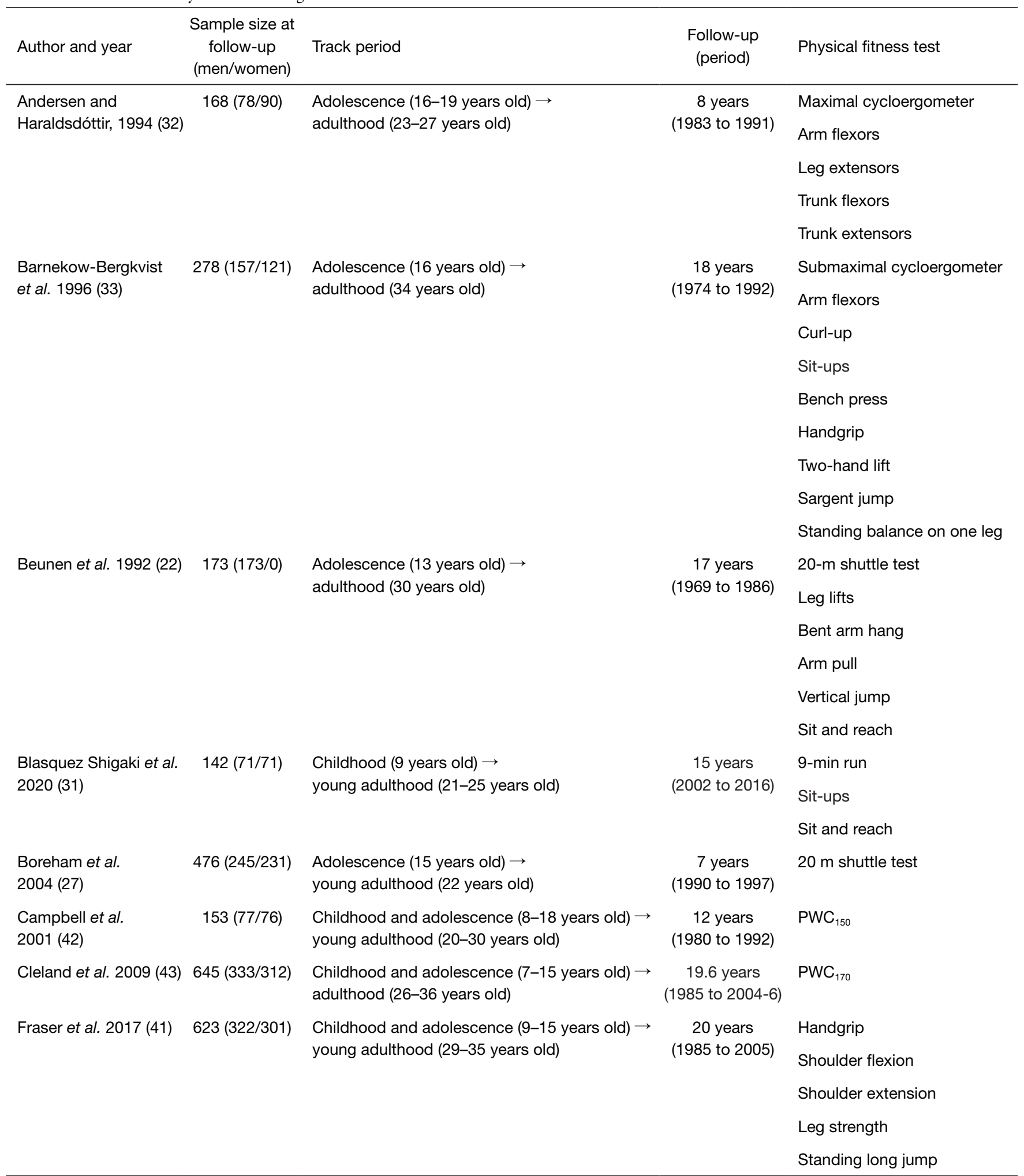

Table 1 (continued) 
Table 1 (continued)

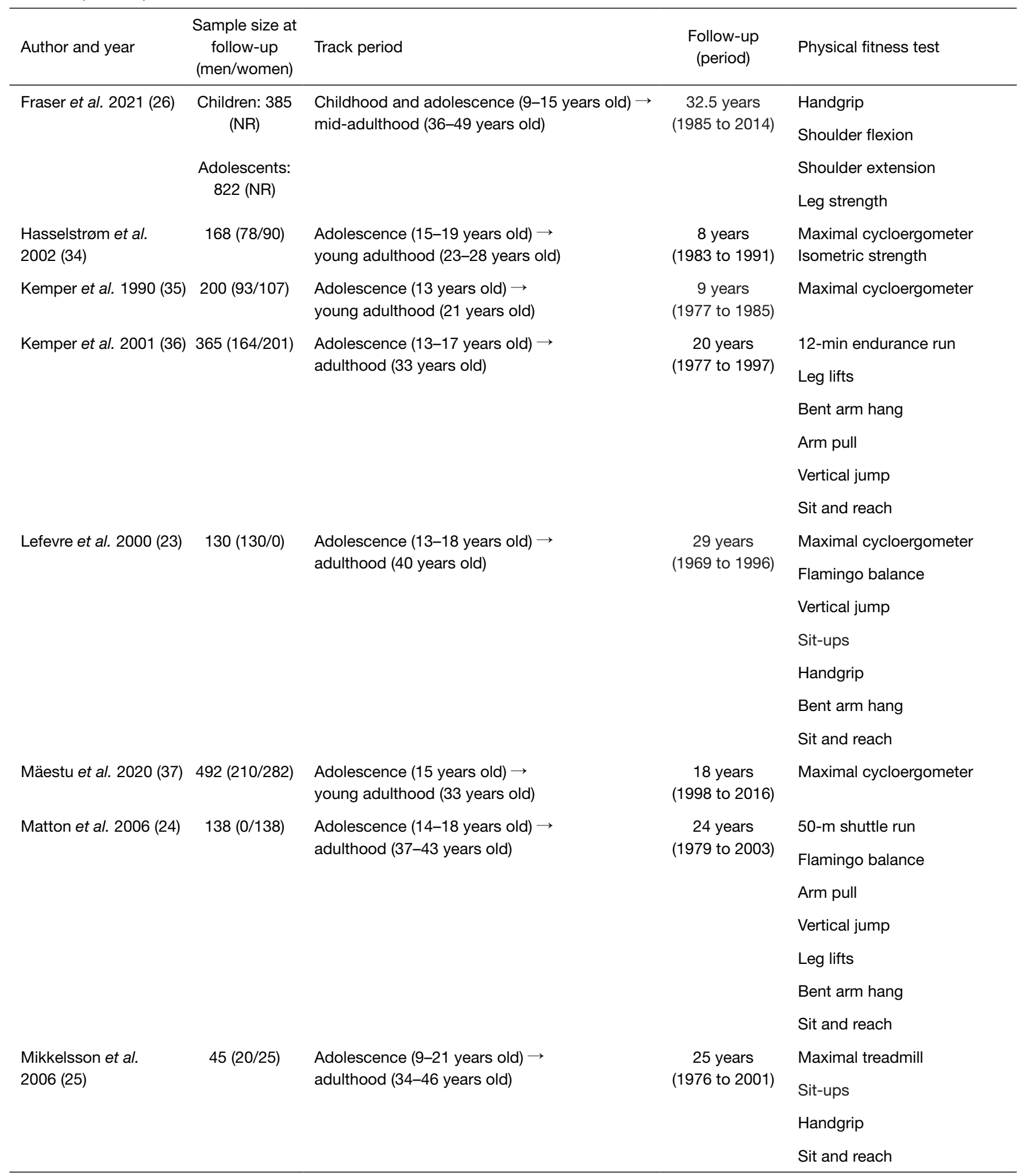

Table 1 (continued) 
Table 1 (continued)

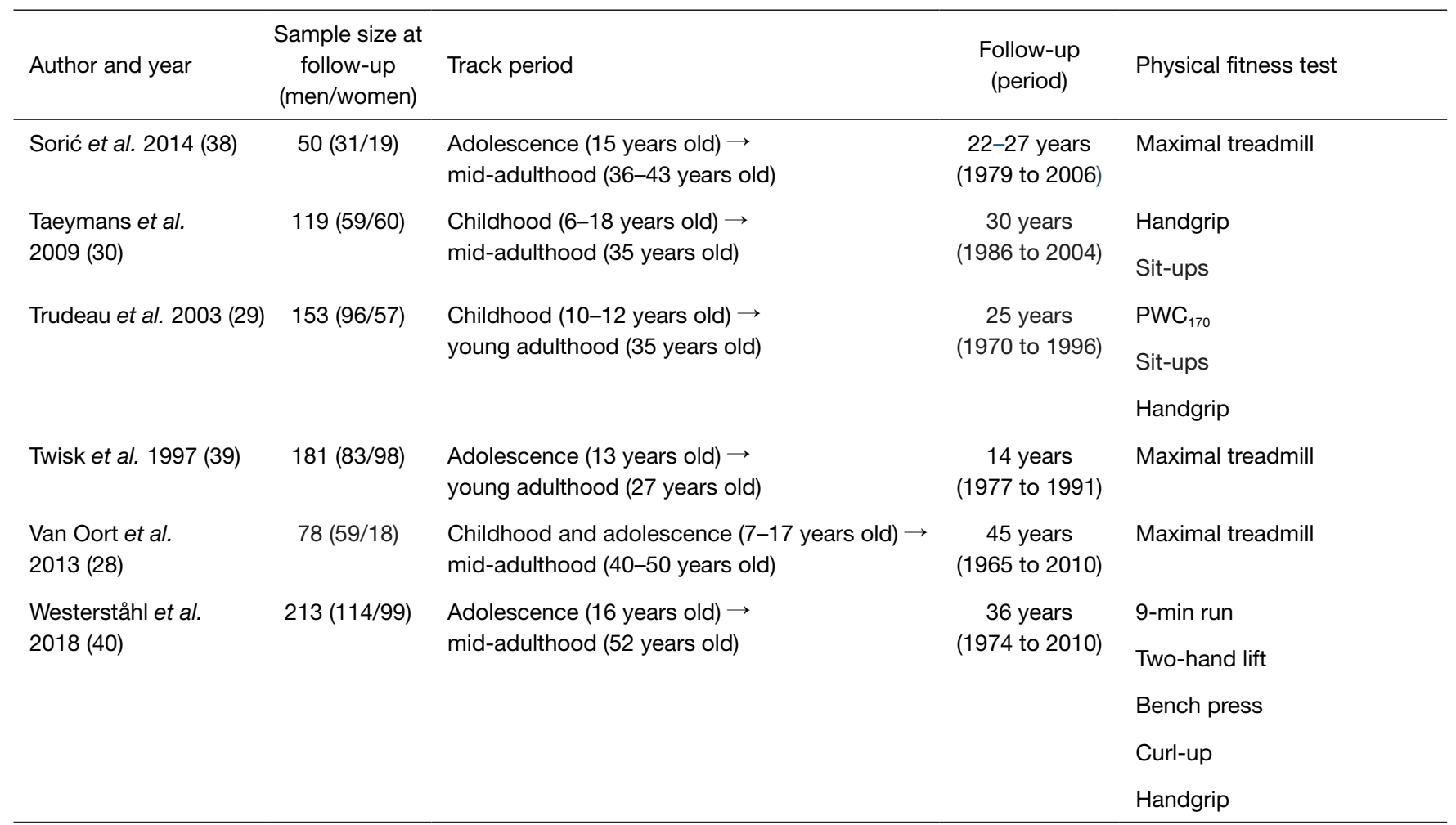

$\mathrm{PWC}_{170}$, physical work capacity at a heart rate of 150 beats per minute; NR, not reported.

Considering the test used, the sit-ups test shows moderate stability ( $\mathrm{r}=0.42 ; 95 \%$ CI: $\left.0.26-0.59 ; \mathrm{I}^{2}=84.5 \%\right)$ and the bent arm hang shows high stability ( $\mathrm{r}=0.67$; $95 \%$ CI: $0.52-0.82$; $\left.\mathrm{I}^{2}=94.1 \%\right)$. Regarding age at baseline, tracking studies from adolescence show a higher correlation coefficient but also moderate ( $\mathrm{r}=0.56$; 95\% CI: $0.41-0.71 ; \mathrm{I}^{2}=94.2 \%$ ).

The meta-regression analysis indicated that the length of follow-up did not moderate this association ( $\beta=-0.008$; 95\% CI: -0.006 to $0.015 ; \mathrm{P}=0.414$ ).

Major asymmetry suggestive of small-study effects was observed (LKF index $=-7.01)$.

The sensitivity analyses showed no modifications in the results after removing one article at a time.

\section{Flexibility}

The tracking for flexibility from childhood and/or adolescence to adulthood was high $(\mathrm{r}=0.69 ; 95 \% \mathrm{CI}$ : $0.58-0.81 ; \mathrm{I}^{2}=92.9 \%$ ) (Figure 2 ), with similar correlation coefficient in men and without inconsistency $(\mathrm{r}=0.68$; 95\% CI: $\left.0.61-0.74 ; \mathrm{I}^{2}=0 \%\right)$ and from adolescence ( $\mathrm{r}=0.71$; 95\% CI: $\left.0.57-0.85 ; \mathrm{I}^{2}=94.4 \%\right)$. Regarding women, only two studies included this fitness component and therefore, no pooled data was analyzed.

The meta-regression analysis showed that the length of follow-up moderates this association, showing higher correlation coefficients with larger follow-up $(\beta=0.017$; 95\% CI; 0.012-0.021; $\mathrm{P}<0.001$ ) (Figure 3).

Major asymmetry suggestive of small-study effects was observed $($ LKF index $=-3.89)$.

The sensitivity analyses showed no modifications in the results after removing one article at a time.

\section{Discussion}

Our study describes the extent of tracking for a range of physical fitness components, from childhood and/or adolescence to adulthood in 3,260 men and 2,937 women from 21 prospective studies. Findings suggest that cardiorespiratory fitness, muscular strength, and muscular endurance have moderate stability from childhood and/ or adolescence to adulthood, independent of test used. This moderate tracking was slightly higher in women than 
Table 2 Items of Quality Assessment Tool for Observational Cohort and Cross-sectional Studies

\begin{tabular}{|c|c|c|c|c|c|c|c|c|c|c|c|c|c|c|c|}
\hline Author, year & 1 & 2 & 3 & 4 & 5 & 6 & 7 & 8 & 9 & 10 & 11 & 12 & 13 & 14 & Total score \\
\hline Andersen and Haraldsdóttir, 1994 (32) & NR & $\sqrt{ }$ & $\checkmark$ & $\sqrt{ }$ & NR & $\sqrt{ }$ & $\sqrt{ }$ & $\sqrt{ }$ & $\sqrt{ }$ & $x$ & $\sqrt{ }$ & $x$ & $x$ & NR & 8 \\
\hline Barnekow-Bergkvist et al. 1996 (33) & $\sqrt{ }$ & $\sqrt{ }$ & NR & $\sqrt{ }$ & NR & $\sqrt{ }$ & $\sqrt{ }$ & $\sqrt{ }$ & $\sqrt{ }$ & $x$ & $\sqrt{ }$ & $x$ & $x$ & NR & 8 \\
\hline Beunen et al. 1992 (22) & $\sqrt{ }$ & $\checkmark$ & $\sqrt{ }$ & $\sqrt{ }$ & NR & $\sqrt{ }$ & $\sqrt{ }$ & $\sqrt{ }$ & $\sqrt{ }$ & $x$ & $\sqrt{ }$ & $x$ & $x$ & NR & 9 \\
\hline Blasquez Shigaki et al. 2020 (31) & $\sqrt{ }$ & $\sqrt{ }$ & $\sqrt{ }$ & $\sqrt{ }$ & NR & $\checkmark$ & $\sqrt{ }$ & $\sqrt{ }$ & $\sqrt{ }$ & $x$ & $\sqrt{ }$ & $x$ & $x$ & NR & 9 \\
\hline Boreham et al. 2004 (27) & $\sqrt{ }$ & $\sqrt{ }$ & $\sqrt{ }$ & $\sqrt{ }$ & NR & $\sqrt{ }$ & $\sqrt{ }$ & $\sqrt{ }$ & $\sqrt{ }$ & $x$ & $\sqrt{ }$ & $x$ & $x$ & NR & 9 \\
\hline Campbell et al. 2001 (42) & $\sqrt{ }$ & $\sqrt{ }$ & NR & $\sqrt{ }$ & NR & $\checkmark$ & $\sqrt{ }$ & $\sqrt{ }$ & $\sqrt{ }$ & $x$ & $\sqrt{ }$ & $x$ & $\sqrt{ }$ & NR & 9 \\
\hline Cleland et al. 2009 (43) & $\sqrt{ }$ & $\sqrt{ }$ & $\sqrt{ }$ & $\sqrt{ }$ & NR & $\checkmark$ & $\sqrt{ }$ & $\sqrt{ }$ & $\sqrt{ }$ & $x$ & $\sqrt{ }$ & $x$ & $x$ & NR & 9 \\
\hline Fraser et al. 2017 (41) & $\sqrt{ }$ & $\sqrt{ }$ & $x$ & $\sqrt{ }$ & NR & $\sqrt{ }$ & $\sqrt{ }$ & $\sqrt{ }$ & $\sqrt{ }$ & $x$ & $\sqrt{ }$ & $x$ & $x$ & NR & 8 \\
\hline Fraser et al. 2021 (26) & $\sqrt{ }$ & $\sqrt{ }$ & NR & $\checkmark$ & NR & $\checkmark$ & $\checkmark$ & $\sqrt{ }$ & $\checkmark$ & $x$ & $\sqrt{ }$ & $x$ & NR & NR & 8 \\
\hline Hasselstrøm et al. 2002 (34) & $\checkmark$ & $\checkmark$ & $\sqrt{ }$ & $\checkmark$ & NR & $\checkmark$ & $\sqrt{ }$ & $\sqrt{ }$ & $\sqrt{ }$ & $x$ & $\checkmark$ & $x$ & $\checkmark$ & NR & 10 \\
\hline Kemper et al. 1990 (35) & $\sqrt{ }$ & $\sqrt{ }$ & NR & $\sqrt{ }$ & NR & $\sqrt{ }$ & $\sqrt{ }$ & $\sqrt{ }$ & $\sqrt{ }$ & $\sqrt{ }$ & $\sqrt{ }$ & $x$ & $x$ & $\mathrm{NR}$ & 9 \\
\hline Kemper et al. 2001 (36) & $\sqrt{ }$ & $\checkmark$ & NR & $\sqrt{ }$ & NR & $\checkmark$ & $\sqrt{ }$ & $\sqrt{ }$ & $\sqrt{ }$ & $\sqrt{ }$ & $\sqrt{ }$ & $x$ & $x$ & NR & 9 \\
\hline Lefevre et al. 2000 (23) & $\sqrt{ }$ & $\sqrt{ }$ & NR & $\sqrt{ }$ & NR & $\sqrt{ }$ & $\checkmark$ & $\sqrt{ }$ & $\sqrt{ }$ & $\sqrt{ }$ & $\sqrt{ }$ & $x$ & $x$ & NR & 9 \\
\hline Mäestu et al. 2020 (37) & $\sqrt{ }$ & $\sqrt{ }$ & $\sqrt{ }$ & $\sqrt{ }$ & NR & $\checkmark$ & $\sqrt{ }$ & $\checkmark$ & $\checkmark$ & $\sqrt{ }$ & $\sqrt{ }$ & $x$ & $\sqrt{ }$ & NR & 11 \\
\hline Matton et al. 2006 (24) & $\sqrt{ }$ & $\sqrt{ }$ & NR & $\sqrt{ }$ & NR & $\checkmark$ & $\checkmark$ & $\sqrt{ }$ & $\sqrt{ }$ & $x$ & $\sqrt{ }$ & $x$ & $x$ & NR & 8 \\
\hline Mikkelsson et al. 2006 (25) & $\checkmark$ & $\sqrt{ }$ & $\sqrt{ }$ & $\sqrt{ }$ & NR & $\checkmark$ & $\checkmark$ & $\sqrt{ }$ & $\checkmark$ & $x$ & $\sqrt{ }$ & $x$ & $x$ & NR & 9 \\
\hline Sorić et al. 2014 (38) & $\checkmark$ & $\sqrt{ }$ & NR & $\checkmark$ & NR & $\checkmark$ & $\checkmark$ & $\sqrt{ }$ & $\checkmark$ & $\checkmark$ & $\sqrt{ }$ & $x$ & $x$ & NR & 9 \\
\hline Taeymans et al. 2009 (30) & $\sqrt{ }$ & $\sqrt{ }$ & NR & $\sqrt{ }$ & NR & $\sqrt{ }$ & $\checkmark$ & $\sqrt{ }$ & $\checkmark$ & $x$ & $\sqrt{ }$ & $x$ & $x$ & NR & 8 \\
\hline Trudeau et al. 2003 (29) & $\checkmark$ & $\checkmark$ & $x$ & $\checkmark$ & NR & $\checkmark$ & $\sqrt{ }$ & $\sqrt{ }$ & $\sqrt{ }$ & $x$ & $\checkmark$ & $x$ & NR & NR & 8 \\
\hline Twisk et al. 1997 (39) & $\sqrt{ }$ & $\checkmark$ & NR & $\checkmark$ & NR & $\checkmark$ & $\sqrt{ }$ & $\sqrt{ }$ & $\sqrt{ }$ & $x$ & $\sqrt{ }$ & $x$ & $\checkmark$ & NR & 9 \\
\hline Van Oort et al. 2013 (28) & $\sqrt{ }$ & $\sqrt{ }$ & NR & $\sqrt{ }$ & NR & $\sqrt{ }$ & $\sqrt{ }$ & $\sqrt{ }$ & $\checkmark$ & $\sqrt{ }$ & $\sqrt{ }$ & $x$ & $x$ & NR & 9 \\
\hline Westerståhl et al. 2018 (40) & $\sqrt{ }$ & $\sqrt{ }$ & NR & $\sqrt{ }$ & NR & $\sqrt{ }$ & $\sqrt{ }$ & $\sqrt{ }$ & $\sqrt{ }$ & $x$ & $\sqrt{ }$ & $x$ & $x$ & NR & 8 \\
\hline
\end{tabular}

$\sqrt{ }$, yes; $\times$, no. NR, not reported.

in men and from adolescence compared to childhood. The stability was also higher in the flexibility component. Therefore, these findings could promote childhood and adolescence physical fitness as a screening tool to help identify youths at high risk of maintaining unfavourable levels of fitness into adulthood. Once high-risk children or adolescents are recognized, their fitness trajectories could and should be improved.

Scientific literature assumed that physical fitness levels during childhood and adolescence is beneficial for health during adulthood. For example, some previous longitudinal studies from a nationwide cohort of one million Swedish men who were examined at 18 years of age suggested that low cardiorespiratory fitness (estimated by a maximal cycloergometer test) and muscular fitness (assessed by knee extension and handgrip tests) were associated with an increased risk of type 2 diabetes (44), myocardial infarction (45), and premature mortality (46) during adulthood. It assumes that children and adolescent physical fitness may have an indirect influence on adult health status by increasing the likelihood of becoming an active adult and have good fitness, which in turn could be beneficial for adult health. Blair et al. (47) also established other two conceptual pathways to explain relationships between physical fitness during childhood and/or adolescence and health during adulthood: through its effect on children and adults health or directly. In this sense, the current study found that both cardiorespiratory fitness and muscular fitness (i.e., muscular strength and endurance) tracking moderate from childhood and/or adolescence to adulthood and high stability for 


\begin{tabular}{|c|c|c|c|c|c|}
\hline Study & & $\begin{array}{l}\text { Correlation }(r) \\
\text { with } 95 \% \mathrm{Cl}\end{array}$ & Follow-up (years) & Sex & Test \\
\hline \multicolumn{6}{|l|}{ Cardiorespiratory fitness } \\
\hline Andersen and Haraldsdóttir, 1994 & & $0.33[0.13,0.53]$ & 8 & Men & Maximal cycloergometer \\
\hline Andersen and Haraldsdóttir, 1994 & & $0.48[0.32,0.64]$ & 8 & Women & Maximal cycloergometer \\
\hline Barnekow-Bergkvist et al. 1996 & & $0.15[-0.00,0.30]$ & 18 & Men & Submaximal cycloergometer \\
\hline Barnekow-Bergkvist et al. 1996 & & $0.40[0.25,0.55]$ & 18 & Women & Submaximal cycloergometer \\
\hline Beunen et al. 1992 & & $0.45[0.33,0.57]$ & 17 & Men & Shuttle run \\
\hline Blasquez Shigaki et al. 2020 & & $0.50[0.32,0.68]$ & 15 & Men & 9-min run \\
\hline Blasquez Shigaki et al. 2020 & & $0.37[0.17,0.57]$ & 15 & Women & 9-min run \\
\hline Campbell et al. 2001 & & $0.24[0.03,0.45]$ & 12 & Men & PWC170 \\
\hline Campbell et al. 2001 & & $0.46[0.28,0.64]$ & 12 & Women & PWC170 \\
\hline Cleland et al. 2009 & & $0.20[0.10,0.30]$ & 19.6 & Men & PWC170 \\
\hline Cleland et al. 2009 & & $0.26[0.16,0.36]$ & 19.6 & Women & PWC170 \\
\hline Hasselstrom et al. 2002 & & $0.35[0.15,0.55]$ & 8 & Men & Maximal cycloergometer \\
\hline Hasselstrom et al. 2002 & & $0.48[0.32,0.64]$ & 8 & Women & Maximal cycloergometer \\
\hline Kemper et al. 1990 & & $0.36[0.18,0.54]$ & 9 & Men & Maximal cycloergometer \\
\hline Kemper et al. 1990 & & $0.46[0.31,0.61]$ & 9 & Women & Maximal cycloergometer \\
\hline Kemper et al. 2002 & & $0.38[0.29,0.47]$ & 20 & Both & 12-min endurance \\
\hline Lefevre et al. 2000 & & $0.58[0.46,0.70]$ & 29 & Both & Maximal cycloergometer \\
\hline Mãestu et al. 2020 & & $0.78[0.75,0.81]$ & 18 & Both & Maximal cycloergometer \\
\hline Matton et al. 2006 & & $0.49[0.36,0.62]$ & 24 & Both & Shuttle run \\
\hline Mikkelsson et al. 2006 & & $0.19[-0.24,0.62]$ & 25 & Men & Maximal treadmill \\
\hline Mikkelsson et al. 2006 & & $0.37[0.02,0.72]$ & 25 & Women & Maximal treadmill \\
\hline Sorić et al. 2014 & & $0.30[0.04,0.56]$ & 24 & Both & Maximal treadmill \\
\hline Trudeau et al. 2003 & - & $0.23[0.04,0.42]$ & 25 & Men & PWC170 \\
\hline Trudeau et al. 2003 & & $0.39[0.17,0.61]$ & 25 & Women & PWC170 \\
\hline Twisk et al. 1997 & & $0.31[0.18,0.44]$ & 14 & Both & Maximal treadmill \\
\hline Van Oort et al. 2013 & & $0.38[0.16,0.60]$ & 45 & Men & Maximal treadmill \\
\hline Van Oort et al. 2013 & & $0.44[0.05,0.83]$ & 45 & Women & Maximal treadmill \\
\hline Westerstáhl et al. 2018 & & $0.28[0.16,0.40]$ & 36 & Both & 9-min run \\
\hline Heterogeneity: $\tau^{2}=0.06, L^{2}=92.73 \%, H^{2}=13.76$ & & $0.38[0.29,0.48]$ & & & \\
\hline \multicolumn{6}{|l|}{ Test of $\theta_{1}=\theta_{;}: Q(27)=371.63, p=0.00$} \\
\hline \multicolumn{6}{|l|}{ Muscular strength } \\
\hline Andersen and Haraldsdóttir, 1994 & & $0.66[0.53,0.79]$ & 8 & Men & Leg extensors \\
\hline Andersen and Haraldsdóttir, 1994 & & $0.69[0.58,0.80]$ & 8 & Women & Leg extensors \\
\hline Barnekow-Bergkvist et al. 1996 & & $0.50[0.38,0.62]$ & 18 & Men & Handgrip \\
\hline Barnekow-Bergkvist et al. 1996 & & $0.56[0.44,0.68]$ & 18 & Women & Handgrip \\
\hline Beunen et al. 1992 & & $0.52[0.41,0.63]$ & 17 & Men & Vertical jump \\
\hline Blasquez Shigaki et al. 2020 & & $0.51[0.33,0.69]$ & 15 & Men & Bench press \\
\hline Blasquez Shigaki et al. 2020 & & $0.58[0.42,0.74]$ & 15 & Women & Bench press \\
\hline Fraser et al. 2017 & & $0.48[0.39,0.57]$ & 20 & Women & Handgrip \\
\hline Fraser et al. 2017 & & $0.47[0.38,0.56]$ & 20 & Men & Standing long jump \\
\hline Hasselstrom et al. 2002 & & $0.19[-0.02,0.40]$ & 8 & Men & Isometric strength \\
\hline Hasselstrom et al. 2002 & & $0.11[-0.09,0.31]$ & 8 & Women & Isometric strength \\
\hline Kemper et al. 2002 & & $0.38[0.29,0.47]$ & 20 & Both & Vertical jump \\
\hline Lefevre et al. 2000 & & $0.82[0.76,0.88]$ & 29 & Both & Leg extensors \\
\hline Matton et al. 2006 & & $0.59[0.48,0.70]$ & 24 & Both & Vertical jump \\
\hline Mikkelsson et al. 2006 & & $0.53[0.19,0.87]$ & 25 & Men & Handgrip \\
\hline Mikkelsson et al. 2006 & & $0.44[0.11,0.77]$ & 25 & Women & Handgrip \\
\hline Trudeau et al. 2003 & & $0.45[0.29,0.61]$ & 25 & Men & Handgrip \\
\hline Trudeau et al. 2003 & & $0.56[0.38,0.74]$ & 25 & Women & Handgrip \\
\hline Westerstáhl et al. 2018 & & $0.44[0.33,0.55]$ & 36 & Both & Bench press \\
\hline Heterogeneity: $\tau^{2}=0.03, l^{2}=87.90 \%, H^{2}=8.26$ & & $0.51[0.43,0.59]$ & & & \\
\hline \multicolumn{6}{|l|}{ Test of $\theta_{1}=\theta_{;}: Q(18)=148.75, p=0.00$} \\
\hline \multicolumn{6}{|l|}{ Muscular endurance } \\
\hline Barnekow-Bergkvist et al. 1996 & & $0.25[0.10,0.40]$ & 18 & Men & Sit-ups \\
\hline Barnekow-Bergkvist et al. 1996 & & $0.51[0.38,0.64]$ & 18 & Women & Sit-ups \\
\hline Beunen et al. 1992 & & $0.46[0.34,0.58]$ & 17 & Men & Bent arm hang \\
\hline Kemper et al. 2002 & & $0.83[0.80,0.86]$ & 9 & Both & Bent arm hang \\
\hline Lefevre et al. 2000 & & $0.79[0.72,0.86]$ & 29 & Both & Bent arm hang \\
\hline Matton et al. 2006 & & $0.56[0.44,0.68]$ & 24 & Both & Bent arm hang \\
\hline Mikkelsson et al. 2006 & & $0.41[0.03,0.79]$ & 25 & Men & Sit-ups \\
\hline Mikkelsson et al. 2006 & & $0.55[0.26,0.84]$ & 25 & Women & Sit-ups \\
\hline Trudeau et al. 2003 & $\rightarrow$ & $0.23[0.04,0.42]$ & 25 & Men & Sit-ups \\
\hline Trudeau et al. 2003 & 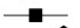 & $0.29[0.05,0.53]$ & 25 & Women & Sit-ups \\
\hline Heterogeneity: $\tau^{2}=0.05,1^{2}=94.47 \%, H^{2}=18.10$ & & $0.50[0.36,0.65]$ & & & \\
\hline \multicolumn{6}{|l|}{ Test of $\theta_{1}=\theta_{1}: Q(9)=162.86, p=0.00$} \\
\hline \multicolumn{6}{|l|}{ Flexibility } \\
\hline Beunen et al. 1992 & & $0.68[0.60,0.76]$ & 17 & Men & Sit and reach \\
\hline Blasquez Shigaki et al. 2020 & & $0.64[0.50,0.78]$ & 15 & Men & Sit and reach \\
\hline Blasquez Shigaki et al. 2020 & & $0.65[0.51,0.79]$ & 15 & Women & Sit and reach \\
\hline Kemper et al. 2002 & & $0.57[0.50,0.64]$ & 9 & Both & Sit and reach \\
\hline Lefevre et al. 2000 & & $0.90[0.87,0.93]$ & 29 & Both & Sit and reach \\
\hline Matton et al. 2006 & & $0.76[0.69,0.83]$ & 24 & Both & Sit and reach \\
\hline Mikkelsson et al. 2006 & & $0.74[0.52,0.96]$ & 25 & Men & Sit and reach \\
\hline Mikkelsson et al. 2006 & & $0.53[0.23,0.83]$ & 25 & Women & Sit and reach \\
\hline Heterogeneity: $\tau^{2}=0.02, l^{2}=92.91 \%, H^{2}=14.10$ & & $0.69[0.58,0.81]$ & & & \\
\hline Test of $\theta_{1}=\theta: Q(7)=98.72, p=0.00$ & & & & & \\
\hline & .5 & 1 & & & \\
\hline
\end{tabular}

Figure 2 Pooled estimated correlation coefficients for fitness tracking from youth to adulthood. PWC $\mathrm{P}_{170}$, physical work capacity at a heart rate of 150 beats per minute. 


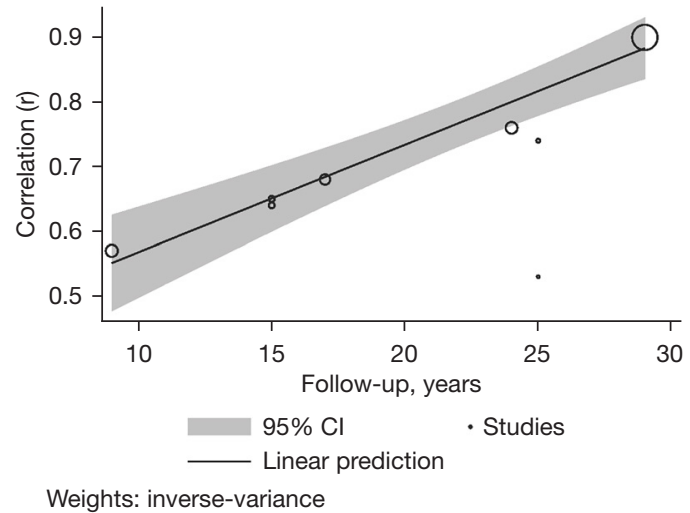

Figure 3 Associations of length of follow-up (years) with correlation coefficients of flexibility tracking from youth to adulthood. The solid line indicates a linear relationship. The size of each data point is proportional to its statistical weight.

flexibility. Therefore, physical capacity seems to be fairly stable, which could be related with fewer health problems as discussed above $(3,44-46)$.

It is interesting to note that the stability of cardiorespiratory fitness is slightly less stable than that of other fitness components. A possible explanation for this finding may be likely a result of the inconsistency of adults to maintain the numerous factors that are known to influence cardiorespiratory fitness (48). We could also speculate that while much activity during young is organized (e.g., school based, sports clubs), by the time the individual reaches early adulthood, practice physical activity is likely to be more a matter of choice (27). It was also suggested that tracking of cardiorespiratory fitness diminishes as the measurement length of follow-up increases (28). This would suggest that youth' cardiorespiratory fitness is more readily maintained over shorter time intervals and their levels could greatly fluctuate with increased length of followup. Otherwise, our meta-regression analyses reported that length of follow-up did not moderate the tracking correlation coefficients, except in the flexibility component.

Regarding muscular fitness, we found that levels of muscular strength and endurance remain relatively moderate stable from childhood or adolescence to adulthood or mid-adulthood. For example, Fraser et al. reported in Australian children and adolescents that those with lower muscular fitness levels had an increased risk of remaining in the same level in adulthood (41) and mid-adulthood (26). These findings highlight young as a potentially key period to promote increased muscular strength and endurance to help encourage favourable levels and healthiest profile into adulthood (5). Another important consideration is that stability seems to be slightly stronger from adolescent than from childhood. A possible explanation for this might be due to a more stable fat free mass and body mass that coincides with later stage puberty compared with pre- and peri-pubertal youth $(22,24)$. Also, women show slightly stronger stability than men, especially in muscular endurance, which may be due to the earlier adrenal androgen production and the higher oestrogen concentration leading to an earlier deceleration of somatic growth in women (49).

The sit and reach test, an indicator of flexibility, showed the highest tracking coefficients from childhood and/ or adolescence to adulthood. Blasquez Shigaki et al. (31) corroborate this finding showing high stability with an intraclass correlation coefficient of 0.64 and 0.65 in men and women, respectively. This may be partly explained by genetic factors (25). Contrary to expectations, metaregression analysis shows stronger stability as years of follow-up increase. Malina (7) corroborated that correlations with flexibility at 30 years tend to increase with age as children progress through adolescence, which could explain our findings.

It is important to bear in mind that children and adolescent differences in the timing and tempo of the growth spurt and sexual maturity may have implications for physical fitness during adulthood. Taeymans et al. (30) showed that adult handgrip strength predictability is low in early maturing boys and late maturing girls. Also, late maturing boys tend to have a favorable strength-to-weight ratio in childhood and at age 35 , showing lower and more stable ratio in all girls groups. In contrast, Beunen et al. (22) show that an advanced biological maturation was related to lower adults' performance for the arm pull and vertical jump tests. This finding is consistent with that of Lefevre et al. (23) who suggest that performance advantage of the early maturing boys assessed during adolescence revers to a disadvantage or disappear at 30 years old.

The generalisability of these results is subject to certain limitations. First, correlations coefficients simply show an association between the past and the present, and therefore, do not predict the future and do not establish a cause-effect order (7). Second, interage age correlations coefficients need to be viewed in the context of healthy lifestyle changes over the past generations. For example, lifestyle changes over the past two generations include reduced levels of physical activity (50) (e.g., reduced active commuting to 
school) and fitness (51), increased screen time (52), among others. It seems possible that these changes could explain large heterogeneity between studies and correlation coefficients, since several studies carried out its followup assessment before year $2000(22,23,27,29,34,36-40,42)$ and others after $2000(24-26,28,30-33,35,41,43)$. Finally, differences in the type of tests, length of follow-up, age at baseline, and different statistical models taken could also explain heterogeneity and inconsistency between correlation coefficients.

\section{Conclusions}

This study has shown that physical fitness is fairly stable from childhood and/or adolescence to adulthood. Given that low levels of cardiorespiratory and muscular fitness in adulthood are related with several chronic diseases, intervention programs for physical fitness training should be planned early. Therefore, our data warrant further research into whether early intervention designed at modifying low youth physical fitness levels could potentially reduce these future chronic diseases (3).

\section{Acknowledgments}

Funding: None.

\section{Footnote}

Reporting Checklist: The authors have completed the PRISMA reporting checklist. Available at https:// tp.amegroups.com/article/view/10.21037/tp-21-507/rc

Conflicts of Interest: All authors have completed the ICMJE uniform disclosure form (available at https://tp.amegroups. com/article/view/10.21037/tp-21-507/coif). AGH serves as an unpaid editorial board member of Translational Pediatrics from August 2021 to July 2023. The other authors have no conflicts of interest to declare.

Ethical Statement: The authors are accountable for all aspects of the work in ensuring that questions related to the accuracy or integrity of any part of the work are appropriately investigated and resolved.

Open Access Statement: This is an Open Access article distributed in accordance with the Creative Commons Attribution-NonCommercial-NoDerivs 4.0 International
License (CC BY-NC-ND 4.0), which permits the noncommercial replication and distribution of the article with the strict proviso that no changes or edits are made and the original work is properly cited (including links to both the formal publication through the relevant DOI and the license). See: https://creativecommons.org/licenses/by-nc-nd/4.0/.

\section{References}

1. Kodama S, Saito K, Tanaka S, et al. Cardiorespiratory fitness as a quantitative predictor of all-cause mortality and cardiovascular events in healthy men and women: a metaanalysis. JAMA 2009;301:2024-35.

2. García-Hermoso A, Cavero-Redondo I, RamírezVélez R, et al. Muscular Strength as a Predictor of AllCause Mortality in an Apparently Healthy Population: A Systematic Review and Meta-Analysis of Data From Approximately 2 Million Men and Women. Arch Phys Med Rehabil 2018;99:2100-13.e5.

3. Ross R, Blair SN, Arena R, et al. Importance of Assessing Cardiorespiratory Fitness in Clinical Practice: A Case for Fitness as a Clinical Vital Sign: A Scientific Statement From the American Heart Association. Circulation 2016;134:e653-99.

4. Ortega FB, Ruiz JR, Castillo MJ, et al. Physical fitness in childhood and adolescence: a powerful marker of health. Int J Obes (Lond) 2008;32:1-11.

5. García-Hermoso A, Ramírez-Campillo R, Izquierdo M. Is Muscular Fitness Associated with Future Health Benefits in Children and Adolescents? A Systematic Review and Meta-Analysis of Longitudinal Studies. Sports Med 2019;49:1079-94.

6. García-Hermoso A, Ramírez-Vélez R, García-Alonso Y, et al. Association of Cardiorespiratory Fitness Levels During Youth With Health Risk Later in Life: A Systematic Review and Meta-analysis. JAMA Pediatr 2020;174:952-60.

7. Malina RM. Physical activity and fitness: pathways from childhood to adulthood. Am J Hum Biol 2001;13:162-72.

8. Janssen I, Leblanc AG. Systematic review of the health benefits of physical activity and fitness in school-aged children and youth. Int J Behav Nutr Phys Act 2010;7:40.

9. National Institute of Health. Study Quality Assessment Tools. Available online: https://www.nhlbi.nih.gov/healthtopics/study-quality-assessment-tools

10. Hardy RJ, Thompson SG. A likelihood approach to metaanalysis with random effects. Stat Med 1996;15:619-29.

11. Malina RM. Tracking of physical activity and physical 
fitness across the lifespan. Res Q Exerc Sport 1996;67:S48-57.

12. Higgins JP, Thompson SG, Deeks JJ, et al. Measuring inconsistency in meta-analyses. BMJ 2003;327:557-60.

13. Higgins JP, Thompson SG. Quantifying heterogeneity in a meta-analysis. Stat Med 2002;21:1539-58.

14. Furuya-Kanamori L, Barendregt JJ, Doi SAR. A new improved graphical and quantitative method for detecting bias in meta-analysis. Int J Evid Based Healthc 2018;16:195-203.

15. Andersen LB. Changes in physical activity are reflected in changes in fitness during late adolescence. A 2-year followup study. J Sports Med Phys Fitness 1994;34:390-7.

16. Fleg JL, Morrell CH, Bos AG, et al. Accelerated longitudinal decline of aerobic capacity in healthy older adults. Circulation 2005;112:674-82.

17. Fortier MD, Katzmarzyk PT, Malina RM, et al. Sevenyear stability of physical activity and musculoskeletal fitness in the Canadian population. Med Sci Sports Exerc 2001;33:1905-11.

18. Andersen LB, Haraldsdóttir J. Tracking of cardiovascular disease risk factors including maximal oxygen uptake and physical activity from late teenage to adulthood. An 8-year follow-up study. J Intern Med 1993;234:309-15.

19. Barnekow-Bergkvist $M$, Hedberg G, Janlert U, et al. Prediction of physical fitness and physical activity level in adulthood by physical performance and physical activity in adolescence--an 18-year follow-up study. Scand J Med Sci Sports 1998;8:299-308.

20. Beunen G, Ostyn M, Simons J, et al. Development and tracking in fitness components: Leuven longtudinal study on lifestyle, fitness and health. Int J Sports Med 1997;18 Suppl 3:S171-8.

21. Dwyer T, Magnussen CG, Schmidt MD, et al. Decline in physical fitness from childhood to adulthood associated with increased obesity and insulin resistance in adults. Diabetes Care 2009;32:683-7.

22. Beunen G, Lefevre J, Claessens AL, et al. Age-specific correlation analysis of longitudinal physical fitness levels in men. Eur J Appl Physiol Occup Physiol 1992;64:538-45.

23. Lefevre J, Philippaerts RM, Delvaux K, et al. Daily physical activity and physical fitness from adolescence to adulthood: A longitudinal study. Am J Hum Biol 2000;12:487-97.

24. Matton L, Thomis M, Wijndaele K, et al. Tracking of physical fitness and physical activity from youth to adulthood in females. Med Sci Sports Exerc 2006;38:1114-20.
25. Mikkelsson L, Kaprio J, Kautiainen H, et al. School fitness tests as predictors of adult health-related fitness. Am J Hum Biol 2006;18:342-9.

26. Fraser BJ, Blizzard L, Buscot MJ, et al. Muscular strength across the life course: The tracking and trajectory patterns of muscular strength between childhood and mid-adulthood in an Australian cohort. J Sci Med Sport 2021;24:696-701.

27. Boreham C, Robson PJ, Gallagher AM, et al. Tracking of physical activity, fitness, body composition and diet from adolescence to young adulthood: The Young Hearts Project, Northern Ireland. Int J Behav Nutr Phys Act 2004;1:14.

28. Van Oort C, Jackowski SA, Eisenmann JC, et al. Tracking of aerobic fitness from adolescence to mid-adulthood. Ann Hum Biol 2013;40:547-53.

29. Trudeau F, Shephard RJ, Arsenault F, et al. Tracking of physical fitness from childhood to adulthood. Can J Appl Physiol 2003;28:257-71.

30. Taeymans J, Clarys P, Abidi H, et al. Developmental changes and predictability of static strength in individuals of different maturity: a 30-year longitudinal study. J Sports Sci 2009;27:833-41.

31. Blasquez Shigaki G, L Barbosa CC, Batista MB, et al. Tracking of health-related physical fitness between childhood and adulthood. Am J Hum Biol 2020;32:e23381.

32. Andersen LB, Haraldsdóttir J. Changes in physical activity, maximal isometric strength and maximal oxygen uptake from late teenage to adulthood: an eight-year follow-up study of adolescents in Denmark. Scand J Med Sci Sports 1994;4:19-25.

33. Barnekow-Bergkvist M, Hedberg G, Janlert U, et al. Development of muscular endurance and strength from adolescence to adulthood and level of physical capacity in men and women at the age of 34 years. Scand J Med Sci Sports 1996;6:145-55.

34. Hasselstrøm H, Hansen SE, Froberg K, et al. Physical fitness and physical activity during adolescence as predictors of cardiovascular disease risk in young adulthood. Danish Youth and Sports Study. An eight-year follow-up study. Int J Sports Med 2002;23 Suppl 1:S27-31.

35. Kemper HC, Snel J, Verschuur R, et al. Tracking of health and risk indicators of cardiovascular diseases from teenager to adult: Amsterdam Growth and Health Study. Prev Med 1990;19:642-55.

36. Kemper HC, de Vente W, van Mechelen W, et al. Adolescent motor skill and performance: is physical activity in adolescence related to adult physical fitness? Am 
J Hum Biol 2001;13:180-9.

37. Mäestu E, Harro J, Veidebaum T, et al. Changes in cardiorespiratory fitness through adolescence predict metabolic syndrome in young adults. Nutr Metab Cardiovasc Dis 2020;30:701-8.

38. Sorić M, Jembrek Gostović M, Gostović M, et al. Tracking of BMI, fatness and cardiorespiratory fitness from adolescence to middle adulthood: the Zagreb Growth and Development Longitudinal Study. Ann Hum Biol 2014;41:238-43

39. Twisk JW, Kemper HC, van Mechelen W, et al. Tracking of risk factors for coronary heart disease over a 14-year period: a comparison between lifestyle and biologic risk factors with data from the Amsterdam Growth and Health Study. Am J Epidemiol 1997;145:888-98.

40. Westerståhl M, Jansson E, Barnekow-Bergkvist M, et al. Longitudinal changes in physical capacity from adolescence to middle age in men and women. Sci Rep 2018;8:14767.

41. Fraser BJ, Schmidt MD, Huynh QL, et al. Tracking of muscular strength and power from youth to young adulthood: Longitudinal findings from the Childhood Determinants of Adult Health Study. J Sci Med Sport 2017;20:927-31.

42. Campbell PT, Katzmarzyk PT, Malina RM, et al. Prediction of physical activity and physical work capacity (PWC150) in young adulthood from childhood and adolescence with consideration of parental measures. Am J Hum Biol 2001;13:190-6.

43. Cleland VJ, Ball K, Magnussen C, et al. Socioeconomic position and the tracking of physical activity and cardiorespiratory fitness from childhood to adulthood. Am J Epidemiol 2009;170:1069-77.

44. Crump C, Sundquist J, Winkleby MA, et al. Physical

Cite this article as: García-Hermoso A, Izquierdo M, RamírezVélez R. Tracking of physical fitness levels from childhood and adolescence to adulthood: a systematic review and meta-analysis. Transl Pediatr 2022;11(4):474-486. doi: 10.21037/tp-21-507
Fitness Among Swedish Military Conscripts and LongTerm Risk for Type 2 Diabetes Mellitus: A Cohort Study. Ann Intern Med 2016;164:577-84.

45. Högström G, Nordström A, Nordström P. High aerobic fitness in late adolescence is associated with a reduced risk of myocardial infarction later in life: a nationwide cohort study in men. Eur Heart J 2014;35:3133-40.

46. Ortega FB, Silventoinen K, Tynelius P, et al. Muscular strength in male adolescents and premature death: cohort study of one million participants. BMJ 2012;345:e7279.

47. Blair S, Clark DG, Cureton KJ, et al. Exercise and fitness in childhood: implications for a lifetime of health. In: Gisolfi CV, Lamb DR. editors. Perspectives in Exercise Science and Sports Medicine. Volume 2. Youth, Exercise and Sport. Carmel, IN, USA: Benchmark Press, 1989:401-30.

48. Hawkins S, Wiswell R. Rate and mechanism of maximal oxygen consumption decline with aging: implications for exercise training. Sports Med 2003;33:877-88.

49. Cameron N, Schell L. Human growth and development. Cambridge, MA, USA: Academic Press, 2012.

50. Guthold R, Stevens GA, Riley LM, et al. Global trends in insufficient physical activity among adolescents: a pooled analysis of 298 population-based surveys with 1.6 million participants. Lancet Child Adolesc Health 2020;4:23-35.

51. Tomkinson GR, Lang JJ, Tremblay MS. Temporal trends in the cardiorespiratory fitness of children and adolescents representing 19 high-income and upper middle-income countries between 1981 and 2014. Br J Sports Med 2019;53:478-86.

52. Bucksch J, Sigmundova D, Hamrik Z, et al. International Trends in Adolescent Screen-Time Behaviors From 2002 to 2010. J Adolesc Health 2016;58:417-25. 